\title{
Growth by cannibalization
}

\section{A new calculation of the process of condensation suggests that more attention should be paid to the correlations between neighbouring droplets.}

HERE is as neat a piece of classical physics as one could hope to find. The problem is the apparently simple one of predicting the behaviour of an assembly of droplets such as might be found in a condensing cloud in the atmosphere. Large drops will grow at the expense of smaller drops. The classical problem, of obvious importance in meteorology, is to know how the size distribution changes with time. It is now thirty years since the essentials of the problem were stated and solved by two Soviet physicists, I.M. Lifshitz and V.V. Slyozov. The nub of their result is that, where large droplets grow at the expense of small, the average radius increases with time $(t)$ in proportion to the cube root, $t^{1 / 3}$.

This cube-root dependence on time of the average radius of the droplets in a cloud seems to be borne out by observation, not merely of the sizes of droplets in an atmospheric water cloud but in other comparable situations, the radii of particles crystallizing from a molten metal, for example. But the other features of the size distribution predicted by Lifshitz and Slyozov are not nearly as well established. And now Dr M. Marder from the University of California at Santa Barbara has been able to correct the older arguments in a particularly interesting way (Phys. Rev. Lett. 55, 2953; 1985).

Because the vapour pressure of a small droplet varies inversely with its radius, small droplets are unstable, and will evaporate rather than grow unless the vapour pressure in the immediate environment is greater than that corresponding to equilibrium. This is why cloud formation requires some mechanism of nucleation, and why the environment of a cloud usually manifests a degree of supersaturation with respect to the equilibrium vapour pressure of bulk water at the temperature of cloud formation. But if those conditions are met, it also follows that large droplets will grow at the expense of smaller droplets.

The practical need is to derive quantitative estimates of the preferential growth of larger particles. The process is dominated by diffusion; a shrinking small drop will not be able to continue shrinking if evaporated water vapour accumulates near the drop, increasing the local vapour pressure above equilibrium value. Similarly, a large drop will not be able to continue growing if it should so denude the local environment of water that the vapour pressure is less than the equilibrium value.
So the rate of growth of a particular droplet may be determined by its immediate past history (which determines whether the immediate neighbourhood is enriched by or depleted of water vapour), as well as by the behaviour of nearby droplets which, by shrinking or growing, may add or subtract water vapour from the environment.

The problem is therefore one in kinetic theory, but with the proviso that equilibrium does not apply. The first successful treatment seems to have been given by the two Soviet physicists. Their argument may be summarized as follows. The simplest case is that in which the medium in which the droplets grow is so massively supersaturated that neighbouring drops have no influence on each other and even the effects of surface tension can be neglected. Then, the rate of growth of a particular drop will be determined by the rate at which water vapour can diffuse to reach its surface. In terms of the radius, $R$, the rate of change will be the simple function $(D / R) . Q^{\circ}$, where $D$ is the molecular diffusion constant, $Q^{\circ}$ is the mixing ratio of the water vapour expressed as the ratio of the volume of the water if it were totally condensed to that of the total space occupied and $R$ enters the equation reciprocally because of the geometry of diffusion onto a small supposedly spherical droplet through the surrounding medium.

A missing ingredient from this extreme approximation has the effect of subtracting from $Q^{\circ}$ an amount $d / R$, where $d$ is some quantity proportional to surface tension and the reciprocal of $R$, appearing as a factor. This accords physically with the circumstance that the effect of surface tension is to reduce the rate of growth of drops, the smaller the droplet the more markedly.

The other obviously missing ingredient is some allowance for the extent to which one drop influences its neighbours. First, it is necessary to adjust $Q^{\circ}$ (by subtraction), the volume fraction of the water already condensed, and then to allow for the way in which the growth of a particular droplet is affected by all the behaviour of all the other droplets in the system, by a separate adjustment representing the influence of each of them. Shrinking droplets will add to the availability of material elsewhere, which explains why the rate of change enters linearly in each adjusting term, adding to the availability of material (and thus $Q^{\circ}$ when it is negative). The radius of every other drop, or rather its square, also enters, but the distant effect of each droplet is attenuated in the same way as diffusive effects are invariably attenuated by gaussian factors in respect of both distance and time; in short, the growth of a particular droplet will be more affected by the recent behaviour of other nearby droplets than by the remote (in time) behaviour of distant droplets. The result is a horrid set of nonlinear interlocking equations that nobody would think of solving directly, not merely because solution would be impossible but because the problem is in any case statistical.

Lifshitz and Slyozov dealt with the problem by averaging, on the specific assumption that drops of different random sizes are randomly distributed through the available space. Apart from their conclusion about the average size $r$, they also concluded that the size distribution of growing droplets, strictly a function both of radius and of time, should ultimately be given by a calculable function only of the ratio $R / r$, which of course involves the time as a variable because the average radius $r$ grows with time. Marder's point is that while the cube-root dependence of $r$ has been well confirmed by observation, the size distribution of particles that grow partly by the remote cannibalization of each other usually turns out to be much broader than that calculated.

What can have gone wrong? Marder's answer is simple; Lifshitz and Slyozov had no business to determine the average behaviour of a droplet by assuming that droplets of different sizes may be randomly distributed. On the contrary, Marder says, precisely because a shrinking drop will add preferentially to the growth of its immediate neighbours rather than more distant drops, there will be a tendency for large and small drops to be paired together statistically. By good fortune, the more exact correlation is calculable. The proof of the conclusion is that the calculated size distribution agrees well with data previously gathered for the particlesize distribution in slowly solidifying alloys of $\mathrm{Fe}-\mathrm{Si}$ - Ti and $\mathrm{Ni}-\mathrm{Si}$, both of which are broader than predicted by Lifshitz and Slyozov. More detailed calculations of the evolution of a system of mutually cannibalizing particles can pass through a stage in which there is a distinctly bimodal distribution of size, with one group of smallish particles and another which is much larger.
John Maddox 